\title{
Decay of a bound muon into a bound electron
}

\author{
M. Jamil Aslam*; Andrzej Czarnecki, Anna Morozova, and Guangpeng Zhang \\ Department of Physics, University of Alberta, Edmonton, T6G 2E1, Alberta, Canada. \\ E-mail: maslameualberta.ca
}

\begin{abstract}
A muon bound to a nucleus can decay into an electron that is either free or remains in a bound state. We here consider the latter case, as part of a larger project of characterizing bound muon decays. These studies are motivated by experimental searches for the lepton-flavor-violating $\mu \rightarrow$ $e$ conversions in the field of a nucleus. Here we focus on the hypothetical case of close electron and muon masses. In this case we present a simple analytical formula for the branching ratio and compare it with a previous publication.
\end{abstract}

XXIX International Symposium on Lepton Photon Interactions at High Energies - LeptonPhoton2019 August 5-10, 2019

Toronto, Canada

\footnotetext{
* Speaker.

$\dagger$ Visiting Professor at the University of Alberta during Sabbatical leave from Quaid-i-Azam University, Islamabad, Pakistan. 


\section{Introduction}

This work is motivated by two large experiments under preparation, Mu2e [1] and COMET [2], that plan to search for the very rare muon-electron conversion near a nucleus, $\mu N \rightarrow e N$.

These experiments will extend the search for lepton-flavor non-conservation conducted by MEG at the Paul Scherrer Institute (PSI) [3]. It will complement a new generation of PSI experiments: MEG II and Mu3e [4]. But whereas MEG II will be looking for $\mu \rightarrow e \gamma$ and aiming for a sensitivity of around one exotic process per $10^{13}$ normal muon decays, Mu2e and COMET hope to be sensitive to a broader set of New Physics processes at the level of $10^{-16}-10^{-18}$. Never has a search been conducted for such rare reactions. The conversion is unique because the signal is clean: a monochromatic electron carrying the energy close to the total muon mass.

The key to success is control over backgrounds. The most dangerous are high-energy electrons from the standard muon decay. When a muon decays in isolation, the daughter electron can have at most half of its mass in energy, because at least the other half must be taken by the neutrinos to balance the momentum. But with a nearby nucleus that can absorb the momentum, the electron energy can reach almost the full muon mass and mimic the conversion signal.

Mapping precisely the spectrum of produced electrons is our long-term goal. In the past, we determined the binding effects, including relativistic effects (crucial for the high-energy tail), the nuclear recoil, and the finite size of the nuclear charge distribution [5]. That analysis, based on a numerical solution of the Dirac equation for the muon and the electron, resulted in a prediction of the spectrum in the full energy range.

That study ignored a small region of the final state phase space namely where the daughter electron is emitted with such low energy or so close to the nucleus that it must remain in a bound state. Here we focus on that region.

Before proceeding to a calculation let us note three features of the bound muon decay that distinguish it from a free-particle decay, as shown in Fig. 1. First, the total available energy is
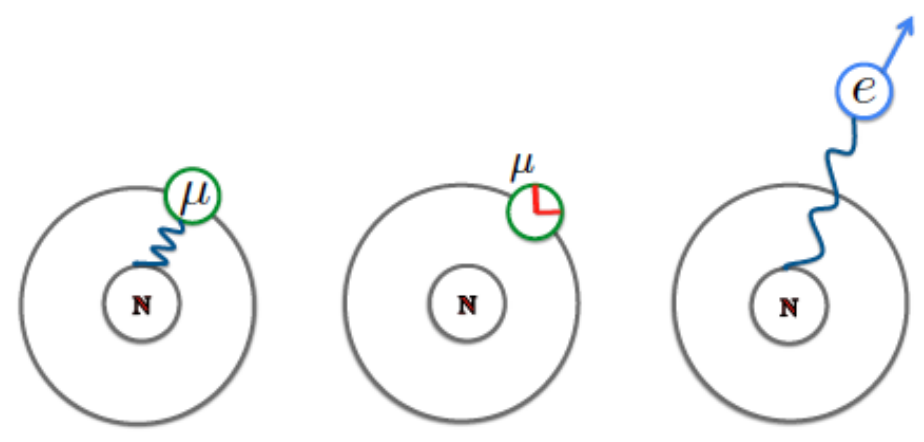

Figure 1: The three features of bound muon decay to an electron, namely, phase space effect (left), time dilation (center) and Coulomb interaction of the daughter electron (right).

decreased by the binding. Second, because the muon is moving, its proper time is shorter than the laboratory time. Finally, the daughter electron is created in the attractive potential of the nucleus. The first two features reduce the decay rate whereas the third one enhances it $[6,7,8,9]$. 
For the typical situation where the electron is in an unbound state, the decay rate is reduced by approximately the time dilation factor [10].

\section{Decay of a bound muon to a bound electron}

We denote a particle $\ell\left(\ell=\mu^{-}\right.$or $\left.e^{-}\right)$bound to a nucleus with atomic number $Z$ by $Z \ell$. The goal of the present contribution is to calculate the rate of the $\left(Z \mu^{-}\right) \rightarrow\left(Z e^{-}\right) \bar{v}_{e} v_{\mu}$ decay for an arbitrary value of $Z$. We will be particularly interested in simplifications in the case of nearly equal muon and electron masses.

We consider the full Dirac wave function for $\mu$ and $e$ bound states and calculate the decay rate in the position space. The ground state (1S) wave function for the bound state in the position space is [11]

$$
\Phi(\vec{r})=\psi_{n=1, j=\frac{1}{2}, \uparrow(\downarrow)}(r, \theta, \phi)=\frac{f(r)}{\sqrt{4 \pi}} u_{+(-)},
$$

where

$$
f(r)=(2 m Z \alpha)^{3 / 2} \sqrt{\frac{1+\gamma}{2 \Gamma(1+2 \gamma)}}(2 m r Z \alpha)^{\gamma-1} \exp (-m r Z \alpha)
$$

and $\gamma=\sqrt{1-(Z \alpha)^{2}}$. In the Dirac representation $u_{+(-)}=\not \rho\left(\begin{array}{c}\phi_{+(-)} \\ 0\end{array}\right)$ with $\rho^{\mu}=\left(\rho^{0}, \vec{\rho}\right)=$ $\left(1, i \frac{1-\gamma}{Z \alpha} \hat{r}\right)$ and $\phi_{+}=\left(\begin{array}{l}1 \\ 0\end{array}\right), \phi_{-}=\left(\begin{array}{l}0 \\ 1\end{array}\right)$.

For the spin sum we use $\sum_{\text {spin }} u \bar{u}=\not \phi \frac{1+\gamma_{0}}{2} \phi^{\prime}$, where $\rho^{\prime \mu}=\left(1,-i \frac{1-\gamma}{Z \alpha} \hat{r}^{\prime}\right)$. The amplitude for the $(Z \mu) \rightarrow(Z e)$ transition can be written as

$$
|\mathscr{M}|^{2}=8 G_{F}^{2} \int d^{3} r d^{3} r^{\prime} \exp \left(i \vec{q} \cdot\left(\vec{r}-\vec{r}^{\prime}\right)\right) \frac{1}{2} \operatorname{Tr}\left[\phi\left(\frac{1+\gamma_{0}}{2}\right) \phi^{\prime} \gamma^{\alpha} P_{L} \phi_{1}^{\prime}\left(\frac{1+\gamma_{0}}{2}\right) \phi_{1} \gamma^{\beta} P_{L}\right],
$$

with $\vec{q}$ denoting the neutrino system momentum which sets the direction of the $z$-axis; and $P_{L}=$ $\frac{1-\gamma_{5}}{2}$. In Eq. (2.3), $\rho_{1}^{\prime \mu} \equiv\left(1, i \frac{1-\gamma}{Z \alpha} \hat{r}^{\prime}\right)=\left(1,-\vec{\rho}^{\prime}\right)$ and $\rho_{1}{ }^{\mu} \equiv\left(1,-i \frac{1-\gamma}{Z \alpha} \hat{r}\right)=(1,-\vec{\rho})$. After taking the trace, doing angular integration and contracting with the neutrino tensor, the expression for the ratio of bound to free muon decay rate becomes

$$
\begin{aligned}
\frac{\Gamma}{\Gamma_{0}}= & \frac{64}{m_{\mu}^{5}(1+\gamma)^{2}} \int_{0}^{E_{\mu}-E_{e}} d|\vec{q}| \vec{q}^{2} \\
& \cdot\left\{\left[q_{0}^{2}-\vec{q}^{2}\right]\left(\frac{1}{3}(2 \gamma+1)^{2}\left\langle\mathscr{J}_{0}\right\rangle^{2}+2\left(1-\gamma^{2}\right)\left\langle\mathscr{J}_{1}\right\rangle^{2}+\frac{2}{3}(1-\gamma)^{2}\left\langle\mathscr{J}_{2}\right\rangle^{2}\right)\right. \\
& \left.+\frac{\vec{q}^{2}}{9}\left(\left(4 \gamma^{2}+4 \gamma+10\right)\left\langle\mathscr{J}_{0}\right\rangle^{2}+4(1-\gamma)^{2}\left\langle\mathscr{J}_{2}\right\rangle^{2}-4\left(1+\gamma-2 \gamma^{2}\right)\left\langle\mathscr{J}_{0}\right\rangle\left\langle\mathscr{J}_{2}\right\rangle\right)\right\},
\end{aligned}
$$

where $\left\langle\mathscr{J}_{k}\right\rangle=\left\langle j_{k}(|\vec{q}| r) f_{e} f_{\mu}\right\rangle$ with $j_{k}(|\vec{q}| r)$ denoting the spherical Bessel function of $k^{\text {th }}$-kind with $k=0,1,2 . f_{e}$ and $f_{\mu}$ are the radial wave functions of electron and muon which can be obtained from Eq. (2.2) by replacing $m$ with $m_{e}$ and $m_{\mu}$, respectively. 
In the equal mass limit we can take the limit $|\vec{q}| r \rightarrow 0$, and hence simplify the spherical Bessel functions $j_{1,2}(|\vec{q}| r) \rightarrow 0$ and $j_{0}(|\vec{q}| r) \rightarrow 1$ in Eq. (2.4). This leads to the simple expression

$$
\frac{\Gamma\left[\left(Z \mu^{-}\right) \rightarrow\left(Z e^{-}\right) \bar{v}_{e} v_{\mu}\right]}{\Gamma_{0}}=\frac{64}{5} \varepsilon^{5} \gamma^{5} \frac{1+\gamma+\gamma^{2}}{3} \quad \varepsilon \ll 1,
$$

where in this limit we have used $\left\langle j_{0}(|\vec{q}| r) f_{e} f_{\mu}\right\rangle^{2} \equiv\left\langle f_{e} f_{\mu}\right\rangle^{2}=\frac{(1+\gamma)^{2}}{4}$ and $\varepsilon=\left(m_{\mu}-m_{e}\right) / m_{\mu}$. The factor $\frac{64}{5} \varepsilon^{5}$ is present also in the free muon decay. Thus the ratio of bound and free decay rates is (note that we are still working to the lowest order in the mass splitting parameter $\varepsilon$ )

$$
\frac{\Gamma\left[\left(Z \mu^{-}\right) \rightarrow\left(Z e^{-}\right) \bar{v}_{e} v_{\mu}\right]}{\Gamma\left[\mu^{-} \rightarrow e^{-} \bar{v}_{e} v_{\mu}\right]}=\gamma^{5} \frac{1+\gamma+\gamma^{2}}{3} \rightarrow 1-3(Z \alpha)^{2}, \quad \varepsilon, Z \alpha \ll 1,
$$

showing a stronger influence of binding than just the time dilation factor, $\sim\left(1-(Z \alpha)^{2} / 2\right)[10]$.

We have checked that our equal mass limit, eq. (2.5), agrees with the previously published result [12]. The next step will be to compare our results for arbitrary muon-to-electron mass ratio.

\section{References}

[1] R. H. Bernstein, The Mu2e Experiment, Front.in Phys. 7, 1 (2019), 1901.11099.

[2] D. Shoukavy, COMET status and plans, EPJ Web Conf. 212, 01006 (2019).

[3] T. Iwamoto, MEG final results and progress towards MEG II, SciPost Phys. Proc. 1, 040 (2019).

[4] P. W. Cattaneo and A. Schöning, MEG II and Mu3e status and plan, EPJ Web Conf. 212, 01004 (2019).

[5] A. Czarnecki, X. Garcia i Tormo, and W. J. Marciano, Muon decay in orbit: spectrum of high-energy electrons, Phys. Rev. D84, 013006 (2011), 1106.4756.

[6] C. Porter and H. Primakoff, The effect of Bohr orbit binding on negative $\mu$-meson $\beta$-decay, Phys. Rev. 83, 849 (1951).

[7] V. Gilinsky and J. Mathews, Decay of bound muons, Phys. Rev. 120, 1450 (1960).

[8] R. Huff, Decay rate of bound muons, Ann. Phys. 16, 288 (1961).

[9] P. Hänggi, R. Viollier, U. Raff, and K. Alder, Muon decay in orbit, Phys. Lett. 51B, 119 (1974).

[10] H. Uberall, Decay of $\mu^{-}$Mesons Bound in the K Shell of Light Nuclei, Phys. Rev. 119, 365-376 (1960).

[11] V. B. Berestetsky, E. M. Lifshitz, and L. P. Pitaevsky, Quantum Electrodynamics, Pergamon, Oxford (1982).

[12] C. Greub, D. Wyler, S. Brodsky, and C. Munger, Atomic alchemy, Phys. Rev. D52, 4028-4037 (1995), hep-ph/9405230. 